\title{
Ground-glass opacity (GGO): a review of the differential diagnosis in the era of COVID-19
}

\author{
Diletta Cozzi ${ }^{1}$ (1) Edoardo Cavigli ${ }^{1}$ (1) $\cdot$ Chiara Moroni ${ }^{1} \cdot$ Olga Smorchkova $^{1} \cdot$ Giulia Zantonelli $^{1} \cdot$ Silvia Pradella $^{1,2}$. \\ Vittorio Miele ${ }^{1}[$
}

Received: 25 February 2021 / Accepted: 7 April 2021 / Published online: 26 April 2021

(c) Japan Radiological Society 2021

\begin{abstract}
Thoracic imaging is fundamental in the diagnostic route of Coronavirus disease 2019 (COVID-19) especially in patients admitted to hospitals. In particular, chest computed tomography (CT) has a key role in identifying the typical features of the infection. Ground-glass opacities (GGO) are one of the main CT findings, but their presence is not specific for this viral pneumonia. In fact, GGO is a radiological sign of different pathologies with both acute and subacute/chronic clinical manifestations. In the evaluation of a subject with focal or diffuse GGO, the radiologist has to know the patient's medical history to obtain a valid diagnostic hypothesis. The authors describe the various CT appearance of GGO, related to the onset of symptoms, focusing also on the ancillary signs that can help radiologist to obtain a correct and prompt diagnosis.
\end{abstract}

Keywords Coronavirus · COVID-19 · Computed tomography $\cdot$ Differential diagnosis · Ground-glass opacity

\section{Introduction}

Ground-glass opacity (GGO) is a non-specific term defined by the Fleischner society as the presence on high-resolution computed tomography (HRCT) of a hazy increase in lung density, not associated with obscuration of the underlying vessels or bronchial walls; if vessels are obscured, the term "consolidation" is preferred [1]. GGO reflects the presence of a various number of lung diseases, such as alveolar collapse, interstitial thickening, or air-space disease: in fact, it is caused by the filling of the alveolar spaces (by cells or fluid) or the thickening of the alveolar walls or interstitium. GGO tends to be difficult to be identified radiographically especially in mild cases: this is because the differential diagnosis of GGO is mainly based on HRCT $[2,3]$. The traditional perception of GGO as a non-specific finding has been superseded by a clinical-radiological evaluation necessary to achieve a valid diagnostic hypothesis [2]. A better approach is to identify the key features of the real underlying

\section{Diletta Cozzi}

dilettacozzi@gmail.com

1 Department of Emergency Radiology, Careggi University Hospital, Largo Brambilla 3, 50134 Florence, Italy

2 SIRM Foundation, via della Signora 2, Milan, Italy disease (such as the onset of symptoms, smoking history, presence/absence of fibrosis, or the distribution in the lung parenchyma) [4, 5]. An organized approach to GGO differential diagnosis is based on the presence of acute versus chronic onset of symptoms (Fig. 1).

In this historical period, especially in the emergency department, the differential diagnosis of GGO is based on the exclusion of the new viral lung infection caused by a novel virus named SARS-CoV-2 (severe acute respiratory syndrome Coronavirus 2) [6-10]. This new coronavirus causes a highly infectious disease, commonly called Coronavirus disease 2019 (COVID-19) [11-17]. The COVID-19 pneumonia is defined as one of the "greatest radiological mimickers" because of the various diseases it may be confused with [18-23]. Typical HRCT pattern in the acute phase of COVID-19 is characterized by the presence of peripheral, subpleural, bilateral, and basal GGOs and consolidations, in some cases also with a super-imposed interlobular/ intralobular septal thickening (the so-called "crazy-paving" pattern) (Fig. 2) [24-28]. Additional findings are vascular enlargement, air bronchograms, and halo or reversed halo signs. Usually, pleural and pericardial effusion, together with lymphadenopathy and cavitations, are rarely observed [29] (Table 1).

The typical GGO appearance in the acute phase of COVID-19 is defined as "rounded-GGO", even if it could 


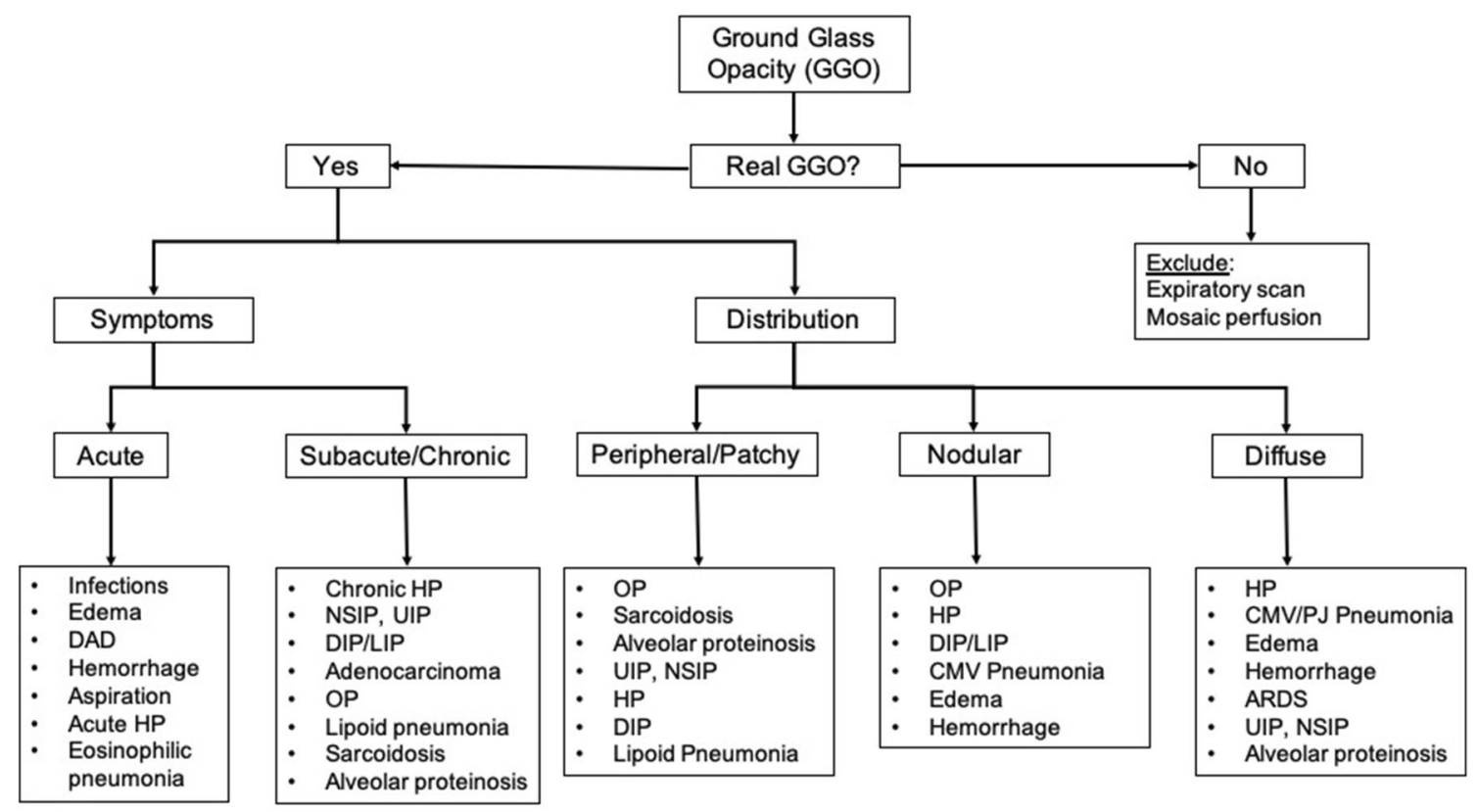

Fig. 1 Algorithmic approach to ground-glass opacities. It is important to exclude causes of false GGO (such as in expiratory scan or in case of mosaic perfusion). $G G O$ ground-glass opacity, $D A D$ diffuse alveolar damage, $H P$ hypersensitivity pneumonitis, $N S I P$ non-specific interstitial pneumonia, UIP usual interstitial pneumonia, DIP desquamative interstitial pneumonia, LIP lymphocytic interstitial pneumonia, $O P$ organizing pneumonia, $C M V$ cytomegalovirus, $P J P$ pneumocystis jirovecii pneumonia, $A R D S$ acute respiratory distress syndrome
Fig. 2 COVID-19 pneumonia. Four cases of GGO in COVID-19 lung involvement in the acute-subacute phase: alterations are mostly peripheral, bilateral, and basal $(\mathbf{a}-\mathbf{c})$. In $\mathbf{d}$, there are halo signs of GGO around the consolidations. COVID-19 coronavirus disease 2019

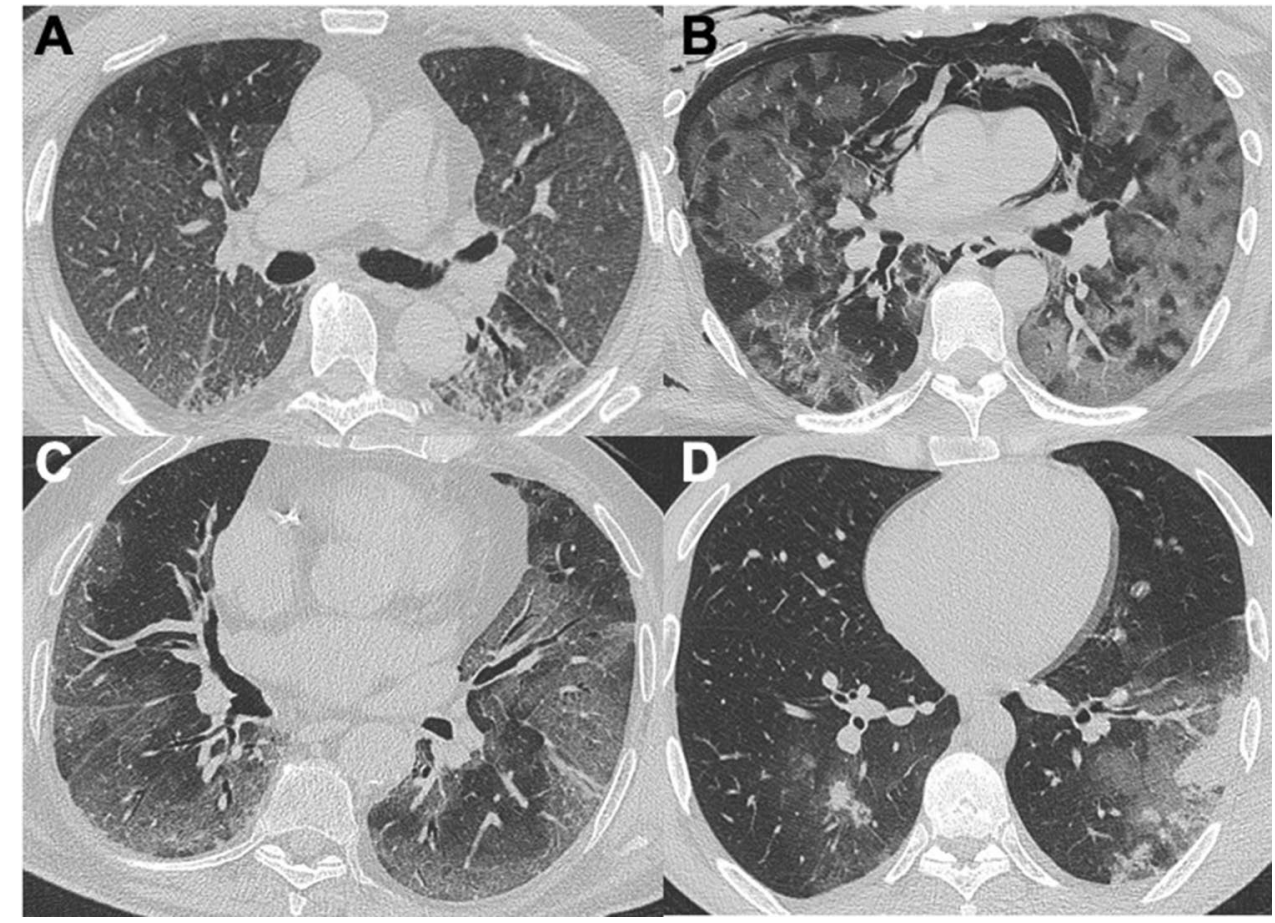

be extensively present as subpleural and bilateral alteration [24-30]. Sometimes, GGO may be the only radiological manifestation of the early lung involvement in the 1-5 days after the onset of symptoms or also in asymptomatic subjects [31-33]. The progressive stage (5-8 days) is characterized by the increase of GGO and crazy-paving appearance until the peak stage (9-13 days), where the alterations tend to be more consolidated. The late stage (more than 14 days) is characterized by a slow decrease of consolidation and GGO, but signs of fibrosis (such as 
Table 1 Typical and atypical HRCT features of COVID-19 infection

HRCT findings in COVID-19 pneumonia

\begin{tabular}{ll}
\hline Typical features & Atypical features \\
\hline Ground-glass opacity & $\begin{array}{c}\text { Centrilobular/nodular } \\
\text { opacity }\end{array}$ \\
Rounded morphology & Cavitation \\
Crazy-paving pattern & Lymphadenopathy \\
Consolidation & Pleural effusion \\
Peripheral distribution & Central distribution \\
Lower distribution & Apical distribution \\
\hline
\end{tabular}

HRCT high-resolution computed tomography, COVID-19 coronavirus disease 2019

subpleural bands, traction bronchiectasis, and architectural distortion) may manifest $[34,35]$. The RSNA has proposed a four categories classification for COVID-19 pneumonia (typical, indeterminate, atypical appearance, or negative for pneumonia) to help radiologists in recognizing the most common findings, decreasing reporting variability, and improving communication with clinicians [36]. Differentiation of COVID-19 pneumonia from other lung diseases is challenging and sometimes very hard [37-40]. The aim of this review is to describe the main differential diagnosis of GGO in the era of COVID-19, based on a clinical-radiological evaluation of various findings (such as the onset of symptoms and the presence of ancillary HRCT signs) that could help radiologists in proposing valid and useful diagnostic hypotheses for an effective multidisciplinary patient's evaluation.

\section{Patients with acute respiratory symptoms}

Pulmonary diseases present acutely within a period of 7-10 days typically. Few pulmonary diseases present in a period ranging from 10 to 30 days and are defined subacute disorders. The most common diseases that present in this time frame and manifest themselves with GGO are similar to the processes associated with water, blood, or pus. Common infection can cause GGO in the acute phase, but also hemorrhage, pulmonary edema, or exacerbations of pre-existing interstitial lung diseases (ILD). In this pandemic period, the HRCT has given fundamental help in the management of patients with acute respiratory symptoms, in particular at the first access to the emergency department. Patients with fever, dyspnea, and non-specific laboratory changes are often evaluated with chest X-ray and HRCT: it is necessary to know the differential diagnoses of GGO, which is one of the most common radiological manifestations of pneumonia from COVID-19.

\section{Infections}

Differentiation of COVID-19 pneumonia from other pulmonary infections could be really challenging, especially from other viral or atypical pneumonia. Viral pneumonia commonly manifests as an interstitial lung involvement [22]. The Influenza virus manifestations were mainly represented by GGOs with consolidation, bilateral patchy consolidation, and ill-defined small nodules [41, 42]. Instead, the $\mathrm{H} 1 \mathrm{~N} 1$ and $\mathrm{H} 5 \mathrm{~N} 1$ forms of influenza, unlike common flu, presented as rapidly progressive pneumonia that very often led to acute respiratory distress syndrome (ARDS). CT imaging features are multifocal consolidations and diffuse areas of GGO, with lymphadenopathy, cavitations, and pleural effusion that are also observed [43, 44]. The respiratory syncytial virus (RSV) more common in children and elderly presents with an airway-centric distribution, with areas of tree-in-bud opacity and bronchial wall thickening, with or without consolidation [45, 46]. Herpes simplex/zoster virus (HSV/VZV) and cytomegalovirus (CMV) pneumonia cause frequently lung diseases in immunocompromised patients: the most frequent radiological findings on $\mathrm{CT}$ examination are interstitial and alveolar infiltrates as bilateral and asymmetric GGO areas, in association with areas of parenchymal consolidation; instead, pleural effusion is more frequent in HSVrelated disease [47, 48]. Differential diagnosis between CMV pneumonia and Pneumocystis Jirovecii pneumonia (PJP) is difficult, especially in the early stages, as the patterns are largely overlapping. Since these pulmonary infections are very common in immunocompromised patients, and the most frequent among infectious complications in patients with AIDS, their differentiation and diagnosis are very important. It should be noted that Pneumocystis Jirovecii can be encountered in mildly immunocompromised patients, such as those with diabetes or with corticosteroid therapies. On CT, the most typical appearance of PJP is bilateral patchy GGO with or without perihilar distribution. CMV shows diffuse micronodules with both centrilobular and random distributions, whereas an apical-perihilar distribution and the presence of mosaic patterns with crazy-paving appearance are more suggestive of PJP [49]. HRCT has a high sensitivity (around 97-98\%) in assessing the presence of abnormalities that may represent pulmonary involvement, although it is not specific in identifying typical viral features (Fig. 3) [50]. Patient's evaluation from a clinical-laboratory point of view is necessary (immunocompromised or not, lymphocyte count, onset symptoms, comorbidities), and sometimes, a bronchoalveolar lavage (BAL) or sputum analysis could be required. In some cases, there might be overlapping between viral and bacterial pneumonia, but, in the latter, there is a higher prevalence of lobar/segmental pneumonia foci or focal/ 


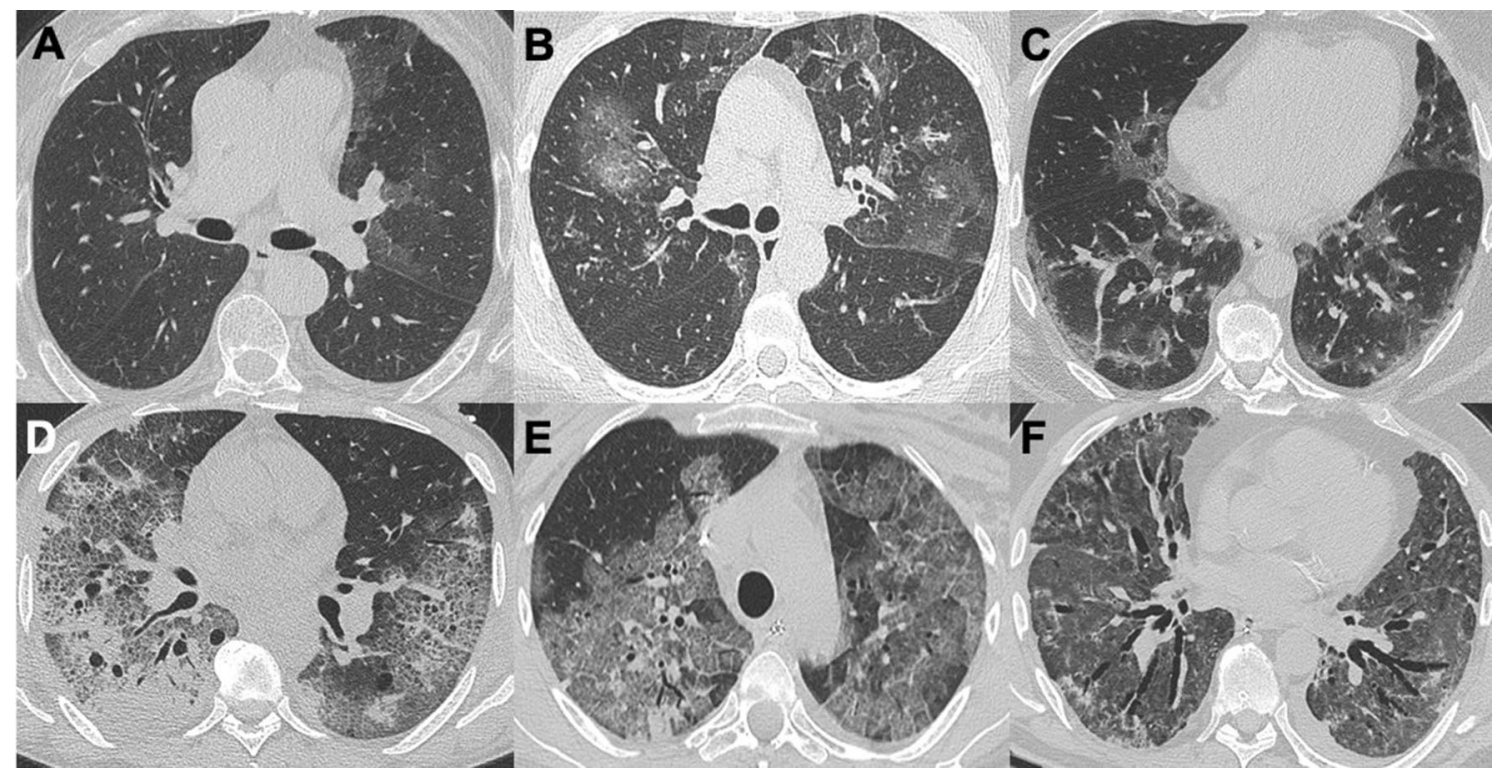

Fig. 3 Infections. $\mathbf{a}$ is a case of early CMV infection with focal GGO in the left upper lobe. $\mathbf{b}$ is a case of EBV pneumonia with mild bilateral GGOs. Figure in $\mathbf{c}$ is a case of pneumonitis caused by Influenza A: HRCT pattern is very similar to COVID-19 pneumonia and differential diagnosis is tricky. Case in $\mathbf{d}$ is a PJP with typical crazy-paving appearance and multiple cysts within the lung. e, $\mathbf{f}$ are two cases

multifocal consolidations [46]. The presence of atypical findings, such as pleural effusion, lymphadenopathies, consolidations, or tree-in-bud appearance in COVID-19 patients, should raise a concern about a co-infection or a super-infection. In fact, a recent meta-analysis reported a viral co-infection rate of $7 \%$ and $3 \%$ for a bacterial one [51].

\section{Hemorrhage}

GGO and consolidations, together with crazy-paving patterns, are typical HRCT manifestations of pulmonary hemorrhage [5]. Lung alterations could be bilateral, focal, or with a patchy distribution, and it depends on the cause of the bleeding: pulmonary contusion or iatrogenic lesion manifest usually as a focal and unilateral GGO, instead hemorrhage caused by vasculitis (Goodpasture syndrome, granulomatosis with polyangiitis, or eosinophilic granulomatosis with polyangiitis), anticoagulation therapy, or cocaine-induced injury manifest as a diffuse lung involvement (Fig. 4) [52-54]. Ancillary signs may be associated, such as halo sign or reversed halo sign, nodules (with or without cavitation) or centrilobular nodules [55]. A targeted medical history is required also in these cases, and serological and clinical findings are essential for differential diagnosis (for example, signs or serological finding of renal impairment could raise the hypothesis of vasculitis). of H1N1 pneumonia with diffuse lung involvement and patient in $\mathbf{f}$ then evolves in ARDS (note the diffuse bronchiectasis and bilateral lung involvement). $C M V$ cytomegalovirus, $E B V$ Epstein-Barr virus, COVID-19 coronavirus disease 2019, PJP pneumocystis jirovecii pneumonia, ARDS acute respiratory distress syndrome

\section{Pulmonary edema}

Hydrostatic pulmonary edema is the most common cause of widespread GGO [2]. Differently from COVID-19, in this case, the presence of central and perihilar predominance of GGO, together with ancillary signs such as pleural effusion, smooth interlobular septal thickening, apical vascular redistribution, cardiomegaly, and peri-bronchial cuffing can help in the differential diagnosis between viral pneumonia and cardiogenic edema (Fig. 5) [56]. GGO is the earliest lung manifestation of cardiogenic pulmonary edema. Instead, non-cardiogenic edema is caused by changes in capillary endothelium, allowing the leakage of fluid in normal hydrostatic pressures (such as in case of aspiration, sepsis, trauma, fat emboli, and illicit drug abuse). In these cases, GGO tends to be more confluent and consolidative, without gravitational distribution, and septal thickening is not a common feature. Consequent diffuse alveolar damage (DAD) reflects acute respiratory distress syndrome (ARDS) and has a specific HRCT pattern: GGO is the most typical finding in DAD; instead, consolidations, fibrosis, and traction bronchiectasis are common features in ARDS [57]. Usually, lung involvement in these cases predominates in the bases, which receive the largest blood volume [5]. Instead, in neurogenic edema (usually seen in patients with brain injury), parenchymal opacities are located in the apices [58]. 
Fig. 4 Hemorrhage. The first case in a is a bilateral alveolar hemorrhage with diffuse perihilar GGOs. Case in $\mathbf{b}$ is a focal parenchymal hemorrhage after cryobiopsy of lung lesion. Hemorrhage with GGO as halo sign in patient with ANCA + vasculitis (c). Finally, $\mathbf{d}$ is a case of "crack-lung", diffuse hemorrhage after inhalation of cocaine. ANCA anti-neutrophil cytoplasmic antibody

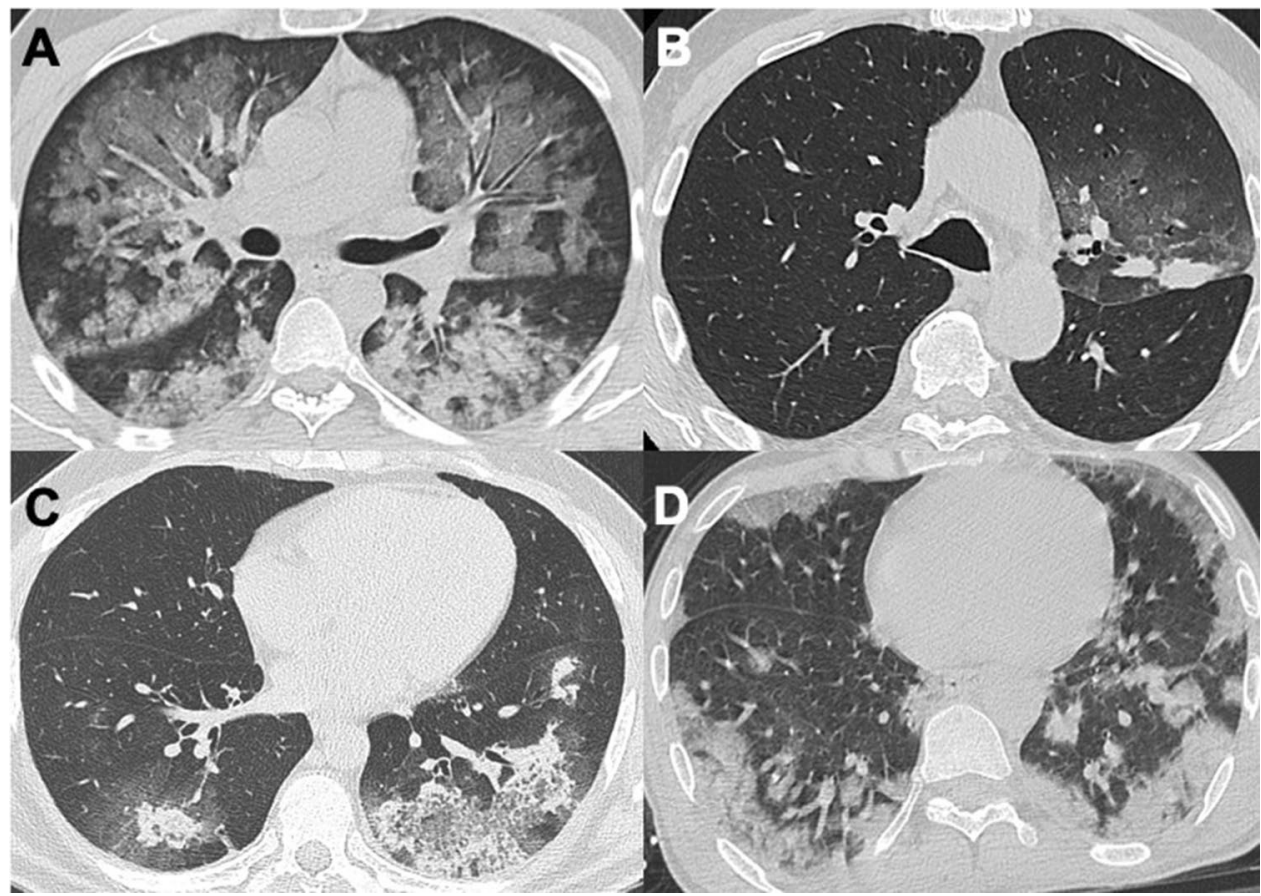

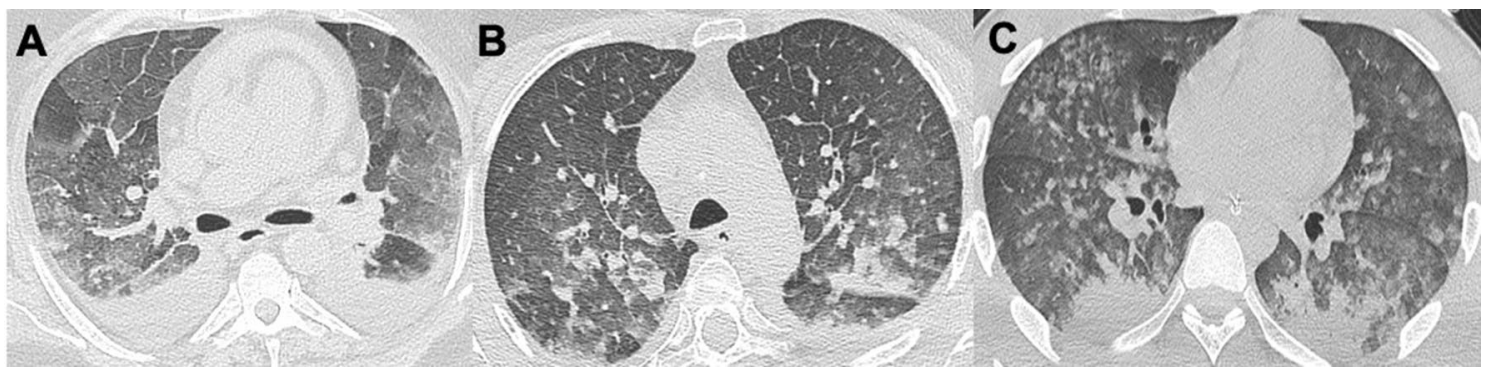

Fig. 5 Pulmonary edema. a, b show two cases of cardiogenic edema: note the pleural effusions, the diffuse and bilateral GGOs with smooth thickening of interlobular septa. $\mathbf{c}$ is a case of acute edema

\section{Acute interstitial pneumonitis (AIP) and acute exacerbation of ILD}

Acute interstitial pneumonitis (AIP-also known as Hamman-Rich syndrome) is a progressive interstitial lung disease (ILD) of unknown etiology that rapidly evolves in DAD/ARDS requiring mechanical ventilation within 7-10 days after the onset of symptoms. AIP has imaging findings similar to ARDS, with extensive areas of symmetrical and bilateral GGO and consolidations (with a predilection towards the lower lobes), with traction bronchiectasis and parenchymal architectural distortion $[5,59]$. The mortality rate is very high within 6 months after presentation [60].

Acute exacerbation of ILD is an acute clinical deterioration in patient with a known (or not) ILD, without a clear reason demonstrable at HRCT such as pulmonary embolism, edema, infection, or left heart failure [61-63]. caused by water aspiration, with diffuse centrilobular nodules surrounded by GGOs, with lung alterations involving mostly the lower posterior lobes

Idiopathic pulmonary fibrosis (IPF) and other ILD can cause these HRCT patterns characterized by diffuse GGO in both lungs on a background of pre-existing areas of pulmonary fibrosis (Fig. 6). Sometimes, an acute exacerbation coexists with super-infection, and it is often associated with poor prognosis and higher mortality rate (between 50 and $90 \%$ in patients admitted to intensivecare units) [64, 65]. Also, COVID-19 infection can cause DAD/ARDS, especially in patients with risk factors such as elder age, chronic cardiac-pulmonary diseases, diabetes, and obesity $[8,16]$. There are no unique data on how often COVID-19 lung damage develops into ARDS, but, if it does, it greatly increases the risk of in-hospital mortality $[19,23,30]$. Usually, ARDS in COVID-19 lung disease develop rapidly within the first 10 days of infection and CT imaging is similar to ARDS from intrapulmonary causes described above. Differential diagnosis could be tricky 


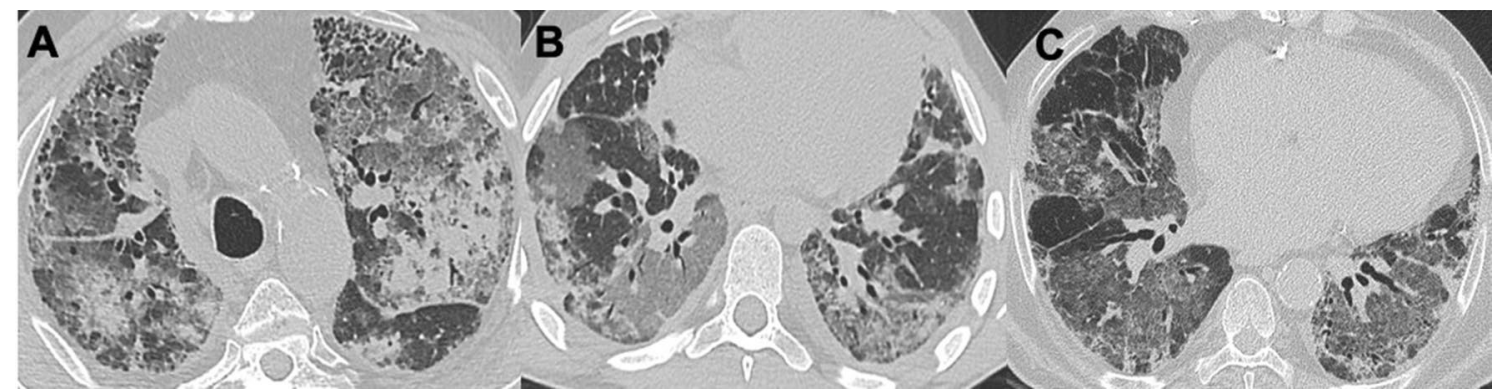

Fig. 6 Acute exacerbation of interstitial lung disease. $\mathbf{a}$ is an acute exacerbation of IPF, with a super-imposed bacterial infection. Patient in $\mathbf{b}$ shows diffuse lung involvement in GVHD after lung transplant in acute lymphatic leukemia. Patient in $\mathbf{c}$ has an exacerbation of NSIP

and, if clinical history and laboratory analysis are not sufficient, BAL or lung biopsy may be required.

\section{Patients with subtle symptoms or asymptomatic}

Several diseases result in GGO in the setting of chronic clinical symptoms (from weeks to months). The spectrum of pathologies is wide and radiological evaluation is based also on the presence of typical parenchymal signs of chronic disease (such as fibrosis and architectural distortion). Moreover, radiologists have to know the duration of symptoms, the smoking history, and other distinguishing clinical features. HRCT gives an advantage in identifying signs of fibrosis and honeycombing, which reveals a chronic process within the lung. The most frequently seen of these pathologies are fibrosing diseases, such as usual interstitial pneumonia (UIP), non-specific interstitial pneumonia (NSIP), hypersensitivity pneumonitis (HP), and respiratory-bronchiolitis ILD (RB-ILD). Moreover, other entities have to be remembered, especially organizing pneumonia (OP), lung involvement in drug toxicities, alveolar proteinosis, and aspiration pneumonia. Neoplastic pathology and pulmonary involvement in sarcoidosis should also not be forgotten.

\section{Interstitial lung diseases}

HRCT findings in ILD may overlap with COVID-19 pneumonia, especially in its subacute-chronic phase. One of the most frequently ILD with diffuse GGO is non-specific interstitial pneumonia (NSIP): together with desquamative interstitial pneumonia (DIP), they both have peripheral GGO in the lower lobes and lack of honeycombing [66, 67]. DIP is included in the group of smoking-related ILD, together with respiratory-bronchiolitis-related ILD (RB-ILD), and may manifest also with mild cystic changes and irregular linear opacities [68]. NSIP occurs more commonly in association with connective-tissue disorders and other HRCT findings are traction bronchiectasis and fine basal reticulation with pattern in antiphospholipid antibody syndrome. IPF idiopathic pulmonary fibrosis, GVHD graft versus-host disease, NSIP non-specific interstitial pneumonia

a typical subpleural sparing (differently from COVID-19 pneumonia) [22, 69]. Instead, a distinctive characteristic of usual interstitial pneumonia (UIP) pattern is the absence of GGO, except in case of an acute exacerbation, as mentioned before (Fig. 7).

Other entities that may present with GGO are lymphocytic interstitial pneumonia (LIP) and interstitial lung abnormalities (ILA). In the first case, there are typical GGOs with diffuse distribution, together with cystic airspaces, lymphadenopathy, and centrilobular nodules [70]. ILA are nowadays increasingly recognized and defined as non-specific interstitial abnormalities involving more than $5 \%$ of any lung zone [71]. Several CT patterns have been described as ILA, including GGO with or without interstitial reticulation, centrilobular nodularity, and traction bronchiectasis: it is important to recognize early fibrosing lung abnormalities, which are more likely to progress and to be associated with mortality [71].

One of the most common subacute-chronic lung diseases is hypersensitive pneumonitis (HP): especially in the nonfibrotic form, it could be mistaken with COVID-19 pneumonia because of its GGO distribution, but the presence of air-trapping and centrilobular GGO nodules both in the upper-middle lung zones are typical for non-fibrotic HP [72]. Air-trapping has a high specificity and sensitivity for HP, but it can be difficult to distinguish the normal increased lung attenuation in the expiration scan, with the diffuse increased attenuation due to GGO (tracheal evaluation has a crucial role in recognizing expiratory $\mathrm{CT}$ scan). In acute HP, HRCT may be normal or shows diffuse GGO; instead, traction bronchiectasis is typical for chronic HP [2]. Differently from the classification based on the time of symptoms' onset, another more recently proposed classification system is based on pathology and distinguishes between fibrotic and non-fibrotic HP, specifying that the latter can itself evolve into a fibrotic form (Fig. 8) [73, 74].

Moreover, acute eosinophilic pneumonia is also characterized with bilateral GGO and consolidations with 
Fig. 7 Interstitial lung disease. NSIP pattern in rheumatoid arthritis with typical GGOs within reticular interstitial involvement, with focal areas of subpleural sparing (a). Another case of NSIP in SSc lung involvement (b). $\mathbf{c}$ is an example of ILA with tiny subpleural GGOs involving more than 5\% of each lobe (arrows). Patient in $\mathbf{d}$ is a case of eosinophilic pneumonia in Churg-Strauss syndrome. NSIP non-specific interstitial pneumonia, $S S c$ systemic sclerosis, ILA interstitial lung abnormalities
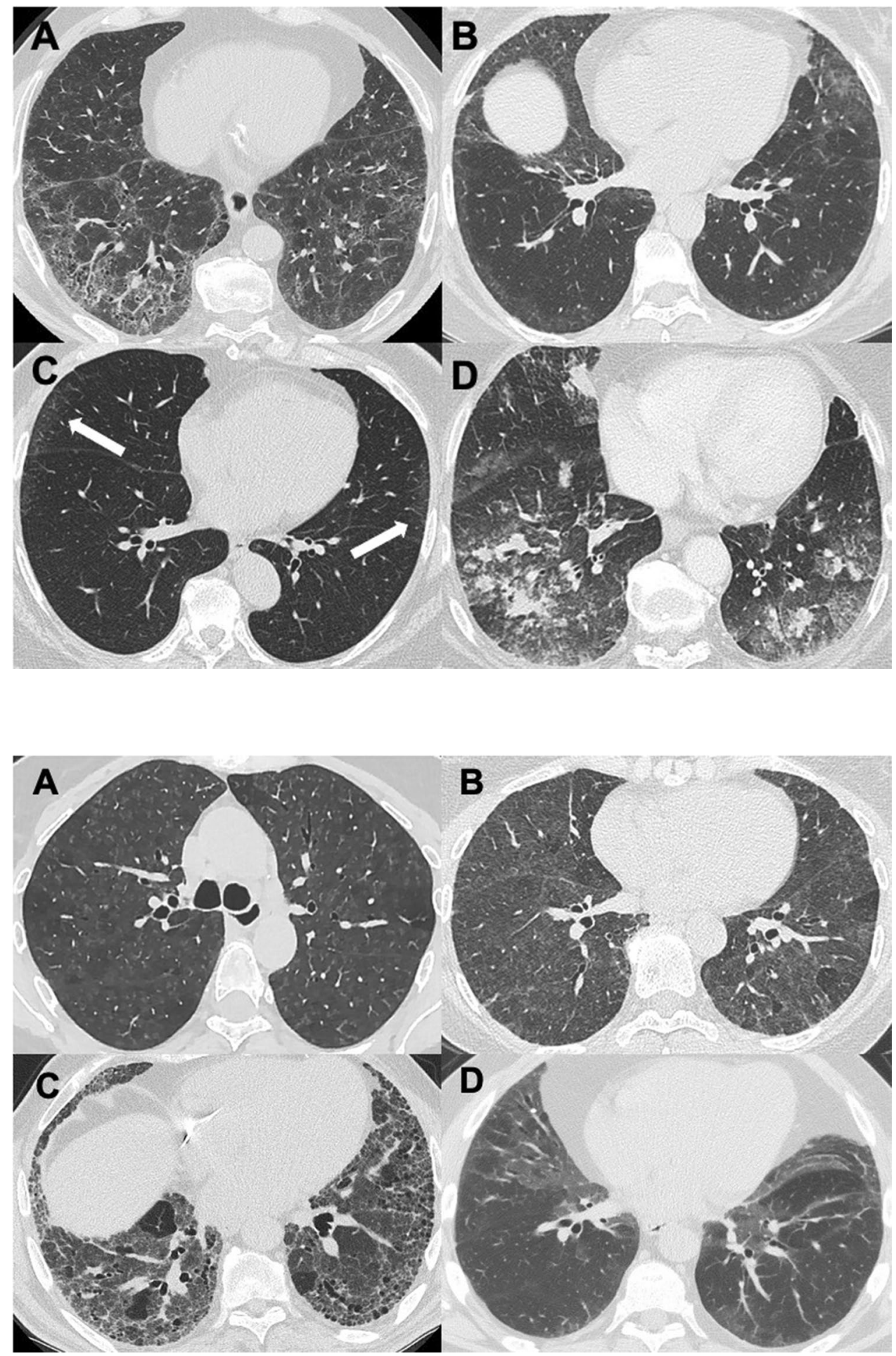

Fig. 8 Interstitial lung disease. $\mathbf{a}$ is an example of non-fibrosing HP (acute), with diffuse GGOs micronodules; instead, a subacute HP is characterized also by focal areas of air-trapping (b). The late stage is fibrosing HP (chronic) with traction bronchiectasis and bilateral basal involvement (c). $\mathbf{d}$ is a case of DIP, the end stage of RB-ILD in a heavy smoker patient, with bilateral and symmetric GGOs. HP hypersensitive pneumonitis, DIP desquamative interstitial lung disease, $R B-I L D$ respiratory-bronchiolitis-related interstitial lung disease interlobular septal thickening and peripheral distribution: unless in COVID-19, the predominance is in the upper-middle lobes and pleural effusion is commonly reported. Diagnosis of eosinophilic pneumonia is tricky, but a characteristic laboratory finding is alveolar and blood eosinophilia [75].

\section{Drug toxicity and OP}

Drug toxicities involving the lung are quite common and often misdiagnosed; clinical manifestations can be variable from an acute form to mild-symptomatic lung disease. Radiologic features and symptoms differ according to the specific 
histopathologic pattern of affection $[69,76]$. In particular, two manifestations that could imitate COVID-19 pneumonia are DAD (in the acute form), HP pattern, and organizing pneumonia (OP) pattern. Some drugs can manifest their toxicity with interstitial pneumonia and differential diagnosis with viral infection could be tricky. OP could be cryptogenic or caused also by infective or other non-infective diseases (post-radiotherapy, vasculitis, collagen, and interstitial lung diseases) [77]. On HRCT, OP shows multifocal GGO and consolidations, usually with a broncho-vascular distribution: reversed halo sign (called also "atoll sign") is typical, even if it is present in only $20 \%$ of cases (Fig. 9) [22, 76]. In COVID-19 pneumonia, reversed halo sign is not a typical feature and it may occur later in disease progression.

\section{Alveolar proteinosis}

Pulmonary alveolar proteinosis (PAP) has some HRCT findings very similar to COVID-19 pneumonia, especially the presence of diffuse and bilateral GGO, crazy-paving appearance, and patchy consolidations. In PAP, one-third of patients are asymptomatic and in $90 \%$ of cases is an idiopathic form [78, 79]. Of course, increasing opacities and acute respiratory symptoms reveal an infectious disease in progression. Since PAP is caused by an abnormal intra-alveolar accumulation of surfactant lipo-proteinaceous material, BAL has both diagnostic and therapeutic role. Moreover, serum anti-GM-CSF (granulocyte-macrophage colonystimulating factor) antibody is useful for diagnosis of autoimmune PAP.

\section{Lipoid pneumonia and aspiration diseases}

Both lipoid and aspiration pneumonia mostly involve lower posterior lobes and typically manifest with GGO and consolidations; sometimes, there is a super-imposed crazypaving pattern and consolidations with adipose density $(<-40 \mathrm{HU})$, especially in case of lipidic accumulation (Fig. 10) [80-82]. In fact, exogenous lipoid pneumonia is caused by the aspiration/inhalation of animal fat or mineral oils (for examples in food, radiographic contrast media, or oil-based medications such as oils for constipation) over an extended period. Symptoms are usually non-specific (dyspnea or cough), and sometimes, lung alterations are detected incidentally $[22,83]$. The knowledge of patients' clinical conditions, pre-existing swallowing disorders or dementia, is fundamental in the differential diagnosis.

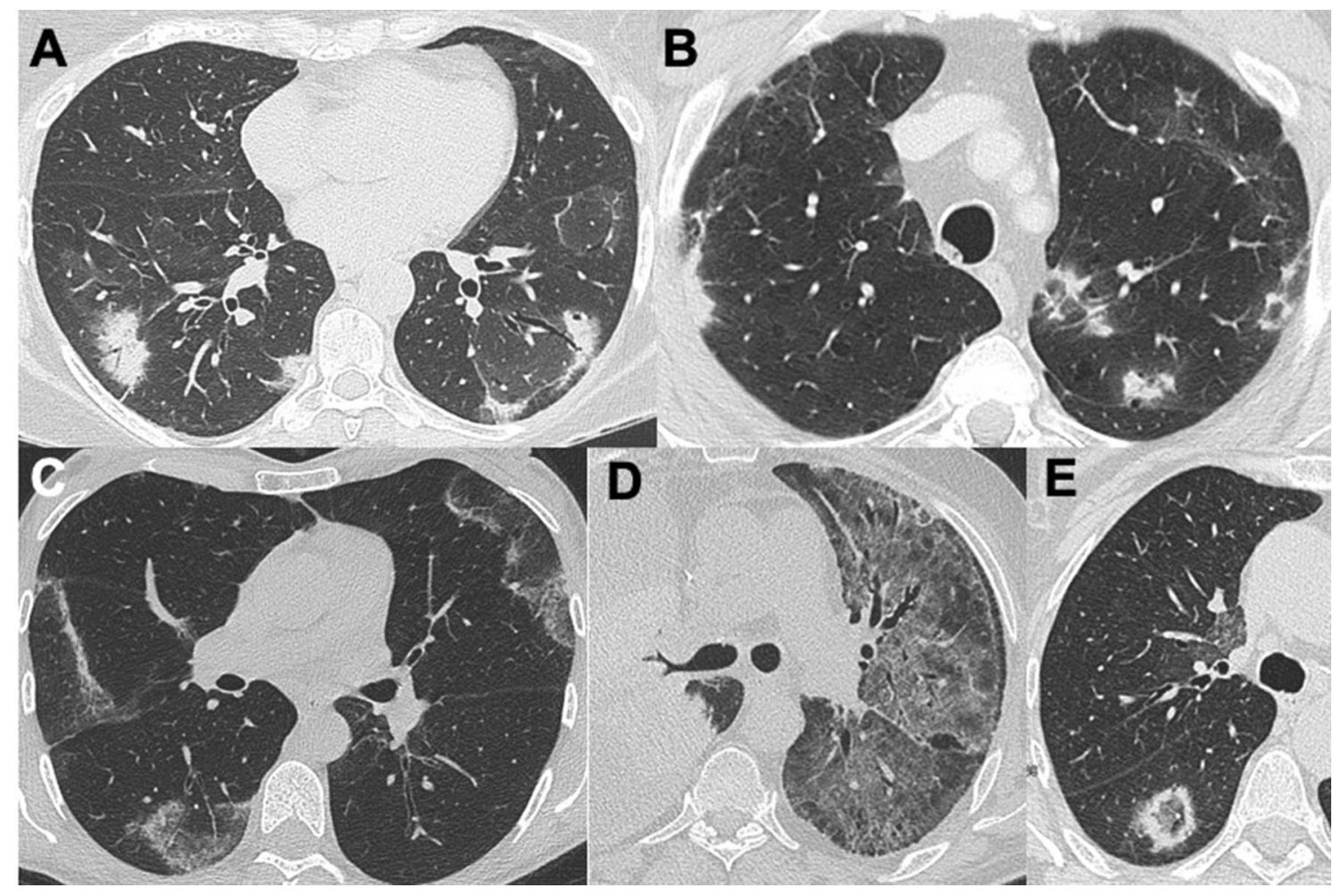

Fig. 9 Drug toxicity and organizing pneumonia. a shows OP pattern after radiation therapy in breast cancer. $\mathbf{b}$ is another OP caused by immunotherapy (Nivolumab) in patient with renal cancer. $\mathbf{c}$ is a case of COP, with diffuse reversed halo signs. $\mathbf{d}$ is a case of interstitial pneumonia after immunotherapy (Pembrolizumab) for right lung cancer and $\mathbf{e}$ is another case of OP with atoll sign. $O P$ organizing pneumonia, $C O P$ cryptogenetic organizing pneumonia 


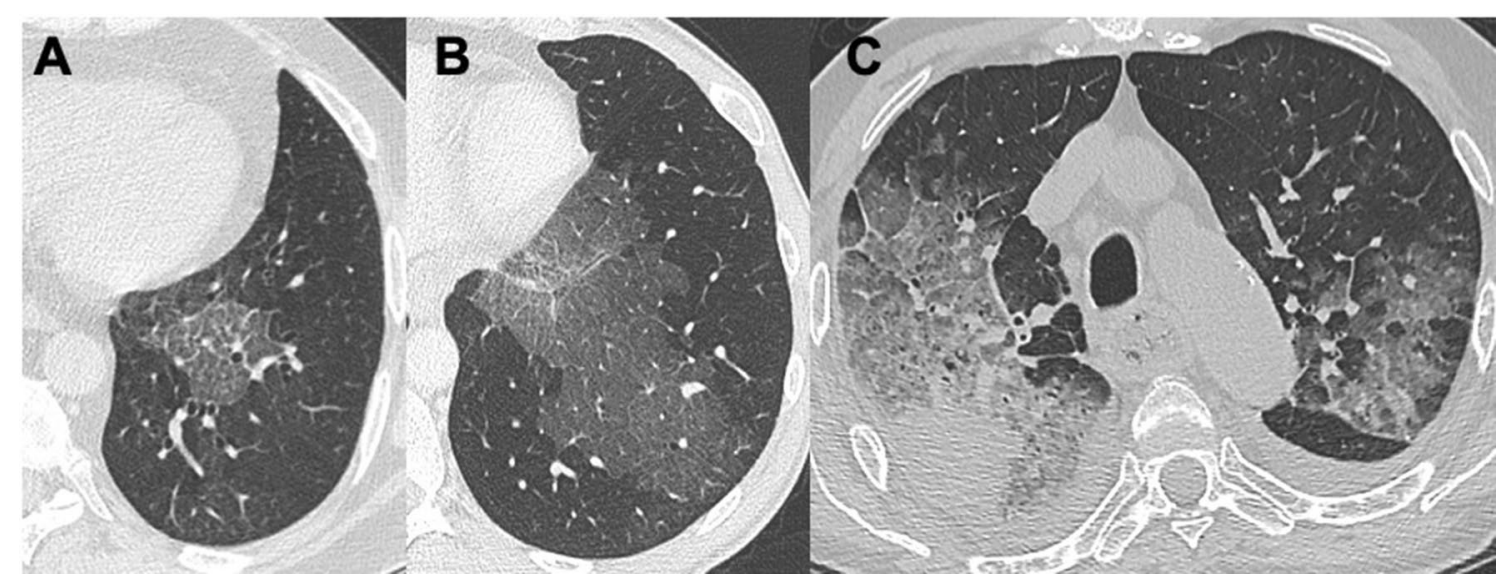

Fig. 10 Aspiration pneumonia. The two cases in a, b show similar GGO areas in lower left lobe, with a super-imposed crazy-paving pattern: the first case $\mathbf{a}$ is a chronic aspiration pneumonia; instead, $\mathbf{b}$ is an example of exogenous lipoid pneumonia in a young patient with chronic inhalation of oily drugs for constipation (confirmed with lung

\section{Cancer}

In COVID-19, pneumonia is described a typical appearance of GGO called "rounded" and, especially if it is a single lesion, neoplastic process should also be considered [22, 84, 85]. Various neoplastic lesions have variable imaging features: in fact, both pre-invasive lesions (for example adenocarcinoma in situ), early- and multifocal adenocarcinoma could manifest as pure GGO nodule, GGO with consolidation, or a solid nodule surrounded by GGO (CT halo sign) [86]. Also, primary pulmonary lymphoma or secondary lesions from adenocarcinoma may manifest as GGO nodules or halo sign (Fig. 11) [87]. CT halo sign could be a manifestation of lepidic tumor cell growth along the alveolar wall in adenocarcinoma, peritumoral hemorrhage, or lymphangitic spread (if associated biopsy). The third case in $\mathbf{c}$ is another example of lipoid pneumonia in elderly, with a rounded consolidation in the right upper lobe and diffuse GGO with crazy-paving in the lower posterior lobes (please note also the dilated esophagus)

with interlobular septal thickening). Ancillary signs usually coexist, such as pleural effusion or hilar-mediastinal lymphadenopathies.

\section{Sarcoidosis}

COVID-19 pneumonia may mimic pulmonary sarcoidosis with atypical HRCT features. In fact, extensive areas of GGO are not common in lung sarcoidosis, but they may be the expression of diffuse coalescent granulomatous micronodules (Fig. 12) [88, 89]. Rarely sarcoidosis may appear as multiple large GGO alterations [4]. The presence of peri-lymphatic nodules with upper lobe predominance and/or mediastinal lymphadenomegaly is suggestive of sarcoidosis and are not typical for COVID-19 pneumonia.

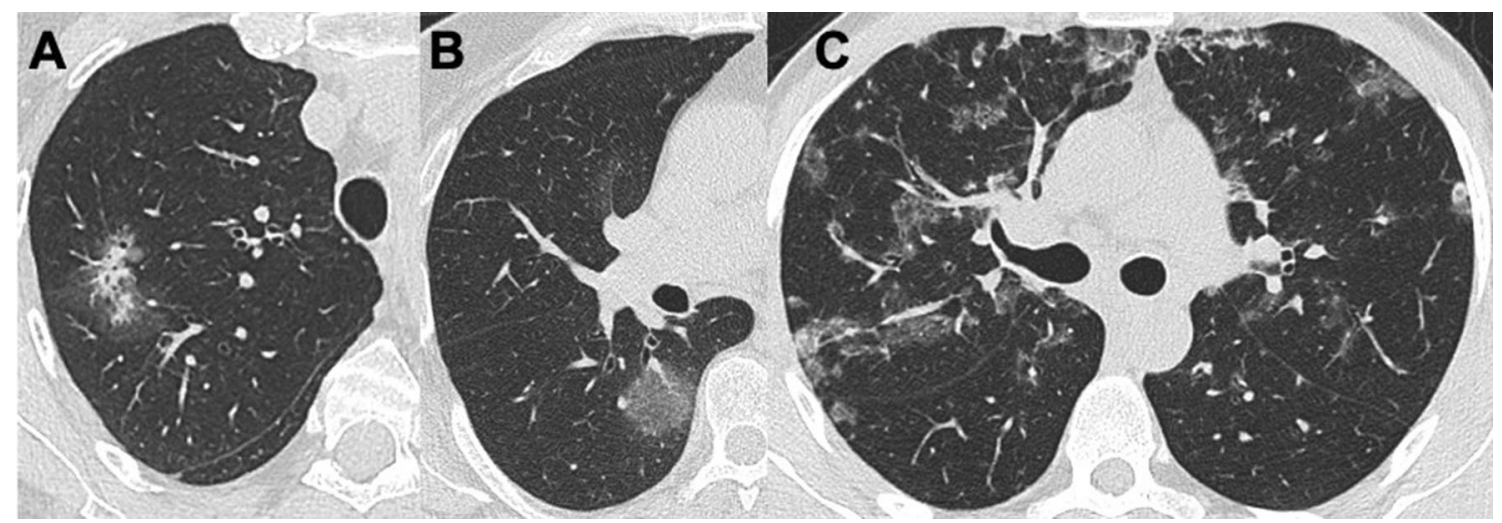

Fig. 11 Cancer. a A lung cancer in the upper right lobe surrounded by GGO halo sign. b An adenocarcinoma with lepidic growth that manifest as a pure GGO nodule. c An example of diffuse bronchial
MALT with bilateral and broncho-vascular involvement. MALT mucose-associated lymphoid tissue 
Fig. 12 Sarcoidosis. a, b Two cases of atypical sarcoidosis pattern with GGO appearance, both caused by diffuse coalescent granulomatous micronodules below HRCT power resolution

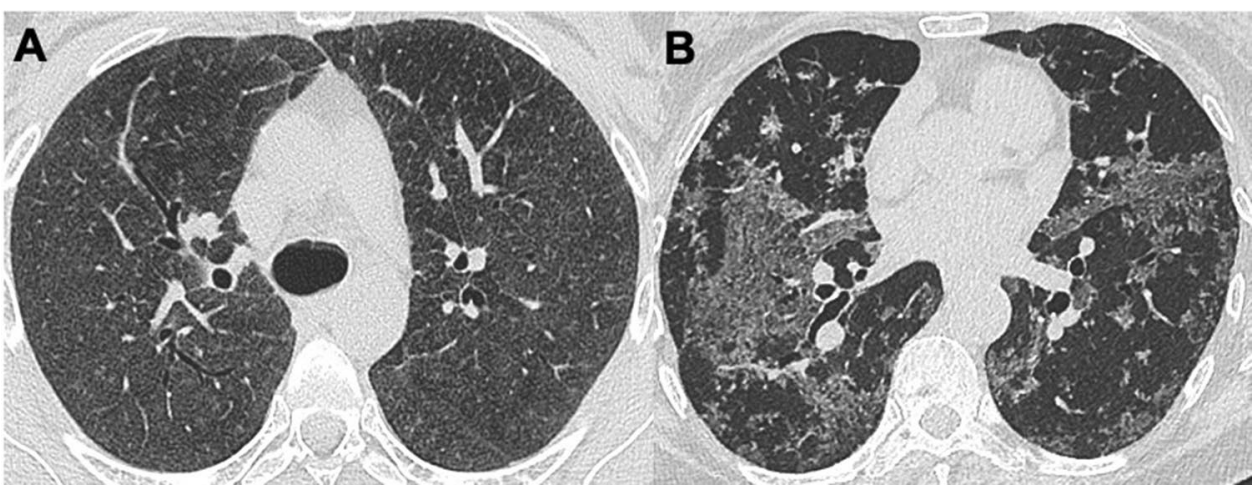

\section{Conclusions}

HRCT is an important diagnostic tool in the management of COVID-19-related pneumonia, with high sensitivity but low specificity. In fact, a broad spectrum of pulmonary parenchymal diseases presents with GGO and may mimic COVID-19 pneumonia. However, ancillary imaging findings and a clinical assessment of the patient, as well as the evaluation of symptom onset, are essential in early diagnosis. Especially, in this pandemic period, a multidisciplinary assessment of the patient is extremely effective in avoiding unnecessary hospitalization or reducing the risk of infection and quarantine, even among health care workers.

Authors contribution All authors contributed to the study conception and design. The first draft of the manuscript was written by DC and EC, and all authors commented on previous versions of the manuscript. All authors read and approved the final manuscript.

\section{Declarations}

Conflict of interest The authors declare that they have no conflict of interest.

Ethical approval This article does not contain any studies with human participants or animals performed by any of the authors.

\section{References}

1. Hansell DM, Bankier AA, MacMahon H, McLoud TC, Muller NL, Remy J. Fleischner society: glossary of terms for thoracic imaging. Radiology. 2008;246(3):697-722.

2. Hewitt MG, Miller WT, Reilly TJ, Simpson S. The relative frequencies of causes of widespread ground-glass opacity: a retrospective cohort. Eur J Radiol. 2014;83:1970-6.

3. Agostini A, Floridi C, Borgheresi A, et al. Proposal of a low-dose, long-pitch, dual-source chest CT protocol on third-generation dual source CT using a tin filter for spectral shaping at $100 \mathrm{kVp}$ for coronavirus disease 2019 (COVID-19) patients: a feasibility study. Radiol Med (Torino). 2020;125(4):365-73.
4. Miller WT, Shah RM. Isolated diffuse ground-glass opacity in thoracic CT: causes and clinical presentations. Am J Roentgenol. 2005;184:613-22.

5. Nowers K, Rasband JD, Berges G, Gosselin M. Approach to ground-glass opacification of the lung. Semin Ultrasound CT MRI. 2002;23(4):302-23.

6. Zhou A, Wang Y, Zhu T, Xia L. CT features of coronavirus disease 2019 (COVID-19) pneumonia in 62 patients in Wuhan, China. Am J Roentgenol. 2020;214:1-8.

7. Giovagnoni A. Facing the COVID-19 emergency: we can and we do. Radiol Med (Torino). 2020;125(4):337-8.

8. Rubin GD, Ryerson CJ, Haramati LB, et al. The role of chest imaging in patient management during the COVID-19 pandemic: a multinational consensus statement from the Fleischner society. Radiology. 2020;296:172-80.

9. Palmisano A, Scotti GM, Ippolito D, et al. Chest CT in the emergency department for suspected COVID-19 pneumonia. Radiol Med (Torino). 2020. https://doi.org/10.1007/s11547-020-01302-y.

10. Kooraki S, Hosseiny M, Myers L, Gholamrezanezhad A. Coronavirus outbreak: what the department of radiology should know. J Am Coll Radiol. 2020. https://doi.org/10.1016/j.jacr.2020.02.008.

11. Zhu N, Zhang D, Wang W, et al. A novel coronavirus from patients with pneumonia in China, 2019. N Engl J Med. 2020. https://doi.org/10.1056/NEJMoa2001017.

12. Albano D, Bruno A, Bruno F, et al. Impact of coronavirus disease 2019 (COVID-19) emergency on Italian radiologists: a national survey. Eur Radiol. 2020. https://doi.org/10.1007/ s00330-020-07046-7.

13. Moroni C, Cozzi D, Albanesi M, et al. Chest X-ray in the emergency department during COVID-19 pandemic descending phase in Italy: correlation with patients' outcome. Radiol Med (Torino). 2021;4:1-8.

14. Borghesi A, Maroldi R. COVID-19 outbreak in Italy: experimental chest $\mathrm{X}$-ray scoring system for quantifying and monitoring disease progression. Radiol Med (Torino). 2020;125(5):509-13.

15. Borghesi A, Zigliani A, Masciullo R, et al. Radiographic severity index in COVID-19 pneumonia: relationship to age and sex in 783 Italian patients. Radiol Med (Torino). 2020;125(5):461-4.

16. Wang C, Horby PW, Hayden FG, Gao GF. A novel coronavirus outbreak of global health concern. Lancet. 2020;395(10223):470-3.

17. Cozzi D, Albanesi M, Cavigli E, et al. Chest X-ray in new Coronavirus disease 2019 (COVID-19) infection: findings and correlation with clinical outcome. Radiol Med (Torino). 2020;125(8):730-7.

18. Neri E, Miele V, Coppola F, Grassi R. Use of CT and artificial intelligence in suspected or COVID-19 positive patients: statement of the Italian society of medical and interventional radiology. Radiol Med (Torino). 2020;125(5):505-8. 
19. Yang W, Sirajuddin A, Zhang X, et al. The role of imaging 2019 in novel coronavirus pneumonia (COVID-19). Eur Radiol. 2020. https://doi.org/10.1007/s00330-020-06827-4.

20. Grassi R, Cappabianca S, Urraro F, et al. Chest CT computerized aided quantification of pneumonia lesions in COVID-19 infection: a comparison among three commercial software. Int J Environ Res Public Health. 2020;17(18):6914.

21. Grassi R, Belfiore MP, Montanelli A, et al. COVID-19 pneumonia: computed-aided quantification of healthy lung parenchyma, emphysema, ground glass and consolidation on chest computed tomography (CT). Radiol Med (Torino). 2020. https://doi.org/10. 1007/s11547-020-01305-9.

22. Duzgun SA, Durhan G, Demirkazik FB, et al. COVID-19 pneumonia: the great radiological mimicker. Insights Imaging. 2020;11:118

23. Carotti M, Salaffi F, Sarzi-Puttini P, et al. Chest CT features of coronavirus disease 2019 (COVID-19) pneumonia: key points for radiologists. Radiol Med (Torino). 2020;125(7):636-46.

24. Ye Z, Zhang Y, Wang Y, Huang Z, Song B. Chest CT manifestations of new coronavirus disease 2019 (COVID-19): a pictorial review. Eur Radiol. 2020. https://doi.org/10.1007/ s00330-020-06801-0.

25. Bianchi A, Mazzoni LN, Busoni S, et al. Assessment of cerebrovascular disease with computed tomography in COVID-19 patients: correlation of a novel specific visual score with increased mortality risk. Radiol Med (Torino). 2020. https://doi.org/10. 1007/s11547-020-01313-9.

26. Machitori A, Noguchi T, Kawata Y, et al. Computed tomography surveillance helps tracking COVID-19 outbreak. Jpn J Radiol. 2020;38(12):1169-76.

27. Rawashdeh MA, Saade C. Radiation dose reduction considerations and imaging patterns of ground glass opacities in coronavirus: risk of over exposure in computed tomography. Radiol Med (Torino). 2020. https://doi.org/10.1007/s11547-020-01271-2.

28. Belfiore MP, Urraro F, Grassi R, et al. Artificial intelligence to codify lung CT in Covid-19 patients. Radiol Med (Torino). 2020;125(5):500-4.

29. Floridi C, Fogante M, Agostini A, et al. Radiological diagnosis of coronavirus disease 2019 (COVID-19): a practical guide. Acta Biomed. 2020;91(8):51-9.

30. Duan Y, Qin J. Pre- and posttreatment chest CT findings: 2019 novel coronavirus (2019-nCoV) pneumonia. Radiology. 2020. https://doi.org/10.1148/radiol.2020200323.

31. Cheng Z, Lu Y, Cao Q, et al. Clinical features and chest CT manifestations of coronavirus disease 2019 (COVID-19) in a singlecenter study in Shangai, China. Am J Roentgenol. 2020;215:1-6.

32. Giannitto C, Sposta FM, Repici A, et al. Chest CT in patients with a moderate or high pretest probability of COVID-19 and negative swab. Radiol Med (Torino). 2020;125(12):1260-70.

33. Ierardi AM, Gaibazzi N, Tuttolomondo D, et al. Deep vein thrombosis in COVID-19 patients in general wards: prevalence and association with clinical and laboratory variables. Radiol Med (Torino). 2021;19:1-7.

34. Fichera G, Stramare R, De Conti G, Motta R, Giraudo C. It's not over until it's over: the chameleonic behavior of COVID-19 over a six-day period. Radiol Med (Torino). 2020;125(5):514-6.

35. Shaw B, Daskareh M, Gholamrezanezhad A. The lingering manifestations of COVID-19 during and after convalescence: update on long term pulmonary consequences of coronavirus disease 2019 (COVID-19). Radiol Med (Torino). 2020. https://doi.org/10.1007/ s11547-020-01295-8.

36. Simpsons S, Kay FY, Abbara S, et al. Radiological society of North America expert consensus statement on reporting chest CT findings related to COVID-19: endorsed by the society of thoracic radiology, the American college of radiology, and RSNA. Radiol Cardiothorac Imaging. 2020;2(2):e200152.
37. Caruso D, Polici M, Zerunian M, et al. Quantitative chest CT analysis in discriminating COVID-19 from non-COVID-19 patients. Radiol Med (Torino). 2020. https://doi.org/10.1007/ s11547-020-01291-y.

38. Lombardi AF, Afsahi AM, Gupta A, Gholamrezanezhad A. Severe acute respiratory syndrome (SARS), Middle East respiratory syndrome (MERS), influenza, and COVID-19, beyond the lungs: a review article. Radiol Med (Torino). 2020. https://doi.org/10. 1007/s11547-020-01311-x.

39. Hosseiny M, Kooraki S, Gholamrezanezhad A, et al. Radiology perspective of coronaavirus disease 2019 (COVID-19): lessons from severe acute respiratory syndrome and middle east respiratory syndrome. Am J Roentgenol. 2020. https://doi.org/10.2214/ AJR.20.22969.

40. Kim H. Outbreak of novel coronavirus (COVID-19): what is the role of radiologists? Eur Radiol. 2020. https://doi.org/10.1007/ s00330-020-06748-2.

41. Leung AN, Gosselin MV, Napper $\mathrm{CH}$, et al. Pulmonary infections after bone marrow transplantation: clinical and radiographic findings. Radiology. 1999;210:699-710.

42. Oikonomou A, Müller NL, Nantel S. Radiographic and high-resolution CT findings of influenza virus pneumonia in patients with hematologic malignancies. Am J Roentgenol. 2003;181(2):507-11.

43. Ajlan AM, Quiney B, Nicolaou S, Müller NL. Swine-origin influenza A (H1N1) viral infection: radiographic and CT findings. Am J Roentgenol. 2009;193(6):1494-9.

44. Qureshi NR, Hien TT, Farrar J, Gleeson FV. The radiologic manifestations of H5N1 avian influenza. J Thorac Imaging. 2006;21(4):259-64.

45. Pierangeli A, Scagnolari C, Antonelli G. Respiratory syncytial virus. Minerva Pediatr. 2018;70(6):553-65. https://doi.org/10. 23736/S0026-4946.18.05312-4 (Epub 2018 Oct 18 PMID: 30334622).

46. Miller WT Jr, Mickus TJ, Barbosa E Jr, Mullin C, Van Deerlin VM, Shiley KT. CT of viral lower respiratory tract infections in adults: comparison among viral organisms and between viral and bacterial infections. Am J Roentgenol. 2011;197(5):1088-95.

47. Chong S, Kim TS, Cho EY. Herpes simplex virus pneumonia: high-resolution CT findings. Br J Radiol. 2010;83(991):585-9.

48. Kim EA, Lee KS, Primack SL, et al. Viral pneumonias in adults: radiologic and pathologic findings. Radiographics. 2002;22:S137-49.

49. Vogel MN, Brodoefel H, Hierl T, Beck R, Bethge WA, Claussen $\mathrm{CD}$, Horger MS. Differences and similarities of cytomegalovirus and pneumocystis pneumonia in HIV-negative immunocompromised patients - thin section CT morphology in the early phase of the disease. Br J Radiol. 2007;80(955):516-23. https://doi.org/ 10.1259/bjr/39696316.

50. Choi H, Qi X, Yoon SH, et al. Extension of coronavirus disease 2019 (COVID-19) on chest CT and implications for chest radiograph interpretation. Radiology. 2020. https://doi.org/10.1148/ ryct.2020200107.

51. Lansbury L, Lim B, Baskaran V, et al. Co-infections in people with COVID-19: a systematic review and meta-analysis. J Infect. 2020;81:266-75.

52. Feragalli B, Martini C, Sperandeo M, et al. The lung in systemic vasculitis: radiological patterns and differential diagnosis. Br J Radiol. 2016;89(1061):20150992.

53. Chung MP, Yi CA, Lee HY, Han J, Lee KS. Imaging of pulmonary vasculitis. Radiology. 2010;255(2):322-41.

54. Kaewlai R, Avery LL, Asrani AV, Novelline RA. Multidetector CT of blunt thoracic trauma. Radiographics. 2008;28(6):1555-70.

55. Marchiori E, Zanetti G, Escuissato GL, et al. Reversed-halo sign: high-resolution CT scan findings in 79 patients. Chest. 2012;141(5):1260-6. 
56. Storto ML, Kee ST, Golden JA, Webb WR. Hydrostatic pulmonary edema: high resolution CT findings. Am J Roentgenol. 1995;165(5):817-20.

57. Goodman LR, Fumagalli R, Tagliabue P, et al. Adult respiratory distress syndrome due to pulmonary and extrapulmonary causes: CT, clinical, and functional correlation. Radiology. 1999;213:545-52.

58. Ell SR. Neurogenic pulmonary edema. A review of the literature and a perspective. Invest Radiol. 1991;26(5):499-505.

59. Kishaba T, Tamaki H, Shimaoka Y, Fukuyama H, Yamashiro S. Staging of acute exacerbation in patients with idiopathic pulmonary fibrosis. Lung. 2014;192:141-9.

60. Ishiguro T, Kobayashi Y, Uozumi R, et al. Viral pneumonia requiring differentiation from acute and progressive diffuse interstitial lung disease. Intern Med. 2019;58(24):3509-19.

61. Richeldi L. Time for prevention of idiopathic pulmonary fibrosis exacerbation. Ann Am Thorac Soc. 2015;12(2):S181-5.

62. Romei C, Tavanti LM, Taliani A, et al. Automated computed tomography analysis in the assessment of idiopathic pulmonary fibrosis severity and progression. Eur J Radiol. 2020;124:108852.

63. Rea G, De Martino M, Capaccio A, et al. Comparative analysis of density histograms and visual scores in incremental and volumetric high-resolution computed tomography of the chest in idiopathic pulmonary fibrosis patients. Radiol Med. 2020. https:// doi.org/10.1007/s11547-020-01307-7.

64. Huie TJ, Olson Al, Cosgrove GP, et al. A detailed evaluation of acute respiratory decline in patients with fibrotic lung disease: aetiology and outcomes. Respirology. 2010;15:909-17.

65. Kishaba T. Acute or subacute progressive interstitial pneumonia. Respir Investig. 2019;57(5):405-7.

66. Tomassetti S, Ryu JH, Piciucchi S, Chilosi M, Poletti V. Nonspecific interstitial pneumonia: what is the optimal approach to management? Semin Respir Crit Care Med. 2016;37(3):378-94.

67. Hellemons ME, Moor CC, von der Thusen J, et al. Dequamative interstitial pneumonia: a systematic review of its features and outcomes. Eur Respir Rev. 2020;29(156):190181.

68. Hidalgo A, Franquet T, Giménez A, et al. Smoking-related interstitial lung diseases: radiologic-pathologic correlation. Eur Radiol. 2006;16(11):2463-70.

69. Elmokadem AH, Batouty NM, Bayoumi D, et al. Mimickers of novel coronavirus disease 2019 (COVID-2019) on chest CT: spectrum of CT and clinical feature. Insight Imaging. 2021;12:12.

70. Lynch DA, Travis WD, Muller NL, et al. Idiopathic interstitial pneumonia: CT features. Radiology. 2005;236(1):10-21.

71. Hatabu H, Hunninghake GM, Richeldi L, Brown KK, et al. Interstitial lung abnormalities detected incidentally on CT: a position paper from the Fleischner society. Lancet Respir Med. 2020;8:726-37.

72. Magee AL, Montner SM, Husain A, et al. Imaging of hypersensitivity pneumonitis. Radiol Clin. 2016;54(6):1033-46.

73. Raghu G, Remy-Jardin M, Ryerson CJ, et al. Diagnosis of hypersensitivity pneumonitis in adults. An official ATS/JRS/ ALAT clinical practice guideline. Am J Respir Crit Care Med. 2020;202(3):e36-69.
74. Kouranos V, Jacob J, Nicholson A, Renzoni E. Fibrotic hypersensitivity pneumonitis: key issues in diagnosis and management. J Clin Med. 2017;6(6):62. https://doi.org/10.1164/rccm. 202005-2032ST.

75. Jeong YJ, Kim KI, Seo IJ, et al. Eosinophilic lung diseases: a clinical, radiologic and pathologic overview. Radiographics. 2007;27(3):617-37.

76. Zare Mehrjardi M, Kahkouee S, Pourabdollah M. Radio-pathological correlations of organizing pneumonia (OP): a pictorial review. Br J Radiol. 2017;90(1071):20160723.

77. Distefano G, Fanzone L, Palermo M, et al. HRCT patterns of drug-induced interstitial lung disease: a review. Diagnostics. 2020;10(4):244.

78. Holbert JM, Costello P, Li W, et al. CT features of pulmonary alveolar proteinosis. Am J Roentgenol. 2001;176(5):1287-94.

79. Suzuki T, Trapnell BC. Pulmonary alveolar proteinosis syndrome. Clin Chest Med. 2016;37(3):431-40.

80. Cozzi D, Bindi A, Cavigli E, et al. Exogenous lipoid pneumonia: when radiologist makes the difference. Radiol Med. 2020. https:// doi.org/10.1007/s11547-020-01230-x.

81. Franquet T, Gimènez A, Roson N, et al. Aspiration diseases: findings, pitfalls and differential diagnosis. Radiographics. 2000;20:673-85.

82. Betancourt S, Martinez-Jimenez S, Rossi S, et al. Lipoid pneumonia: spectrum of clinical and radiologic manifestations. Am J Roentgenol. 2010;194:103-9.

83. Cozzi D, Moroni C, Addeo G, et al. Radiological patterns of lung involvement in inflammatory bowel disease. Gastroenterol Res Pract. 2018;5697846.

84. Meng Y, Liu CL, Cai Q, Shen YY, Chen SQ. Contrast analysis of the relationship between the HRCT sign and new pathologic classification in small ground glass nodule-like lung adenocarcinoma. Radiol Med. 2019;124(1):8-13.

85. Lee HY, Choi YL, Lee KS, et al. Pure ground-glass opacity neoplastic lung nodules: histopathology, imaging, and management. Am J Roentgenol. 2014;202(3):224-33.

86. Gaikwad A, Gupta A, Hare S, et al. Primary adenocarcinoma of lung: a pictorial review of recent updates. Eur J Radiol. 2012;81(12):4146-55.

87. Cozzi D, Dini C, Mungai F, Puccini B, Rigacci L, Miele V. Primary pulmonary lymphoma: imaging findings in 30 cases. Radiol Med. 2019;124(12):1262-9.

88. Criado E, Sanchez M, Ramìrez J, et al. Pulmonary sarcoidosis: typical and atypical manifestations at high-resolution CT with pathologic correlation. Radiographic. 2010;30:1567-86.

89. Cozzi D, Bargagli E, Calabrò AG, Torricelli E, Giannelli F, Cavigli E, Miele V. Atypical HRCT manifestations of pulmonary sarcoidosis. Radiol Med. 2018;123(3):174-84.

Publisher's Note Springer Nature remains neutral with regard to jurisdictional claims in published maps and institutional affiliations. 